Research Article

\title{
Block Preconditioned SSOR Methods for $H$-Matrices Linear Systems
}

\author{
Zhao-Nian Pu and Xue-Zhong Wang \\ School of Mathematics and Statistics, Hexi University, Zhangye, Gansu 734000, China \\ Correspondence should be addressed to Xue-Zhong Wang; mail2011wang@gmail.com \\ Received 9 January 2013; Accepted 12 March 2013 \\ Academic Editor: Hak-Keung Lam
}

Copyright ( 2013 Z.-N. Pu and X.-Z. Wang. This is an open access article distributed under the Creative Commons Attribution License, which permits unrestricted use, distribution, and reproduction in any medium, provided the original work is properly cited.

We present a block preconditioner and consider block preconditioned SSOR iterative methods for solving linear system $A x=b$. When $A$ is an $H$-matrix, the convergence and some comparison results of the spectral radius for our methods are given. Numerical examples are also given to illustrate that our methods are valid.

\section{Introduction}

For the linear system

$$
A x=b,
$$

where $A$ is an $n \times n$ square matrix and $x$ and $b$ are $n$-dimensional vectors. The basic iterative method for solving (1) is

$$
M x^{k+1}=N x^{k}+b, \quad k=0,1, \ldots,
$$

where $A=M-N$ and $M$ is nonsingular. Thus (2) can be written as

$$
x^{k+1}=T x^{k}+c, \quad k=0,1, \ldots,
$$

where $T=M^{-1} N, c=M^{-1} b$.

Let us consider the following partition of $A$ :

$$
A=\left(\begin{array}{cccc}
A_{11} & A_{12} & \cdots & A_{1 m} \\
A_{21} & A_{22} & \cdots & A_{2 m} \\
\vdots & \vdots & \ddots & \vdots \\
A_{m 1} & A_{m 2} & \cdots & A_{m m}
\end{array}\right),
$$

where the blocks $A_{i i} \in C^{n_{i} \times n_{i}}, i=1, \ldots, m$, are nonsingular and $n_{1}+n_{2}+\cdots+n_{m}=n$.

Usually we split $A$ into

$$
A=D-L-U,
$$

where $D=\operatorname{diag}\left(A_{11}, \ldots, A_{m m}\right),-L$ and $-U$ are strictly block lower and strictly block upper triangular parts of $A$, respectively. Let $0<\omega<2$, and

$$
\begin{gathered}
M=\frac{1}{\omega(2-\omega)}(D-\omega L) D^{-1}(D-\omega U), \\
N=\frac{1}{\omega(2-\omega)}((1-\omega) D+\omega L) D^{-1}((1-\omega) D+\omega U) .
\end{gathered}
$$

Then, the iteration matrix of the SSOR method for $A$ is given by

$$
\begin{aligned}
\mathscr{L}_{\omega}= & M^{-1} N \\
= & (D-\omega U)^{-1} D(D-\omega L)^{-1} \\
& \times((1-\omega) D+\omega L) D^{-1} \\
& \times((1-\omega) D+\omega U) .
\end{aligned}
$$

Transforming the original system (1) into the preconditioned form

$$
P A x=P b,
$$


then we can define the basic iterative scheme:

$$
M_{p} x^{k+1}=N_{p} x^{k}+P b, \quad k=0,1, \ldots
$$

where $P A=M_{p}-N_{p}$ and $M_{p}$ is nonsingular. Thus (9) can also be written as

$$
x^{k+1}=T x^{k}+c, \quad k=0,1, \ldots,
$$

where $T=M_{p}^{-1} N_{p}, c=M_{p}^{-1} P b$. Similar to the original system (1), we call the basic iterative methods corresponding to the preconditioned system the preconditioned iterative methods.
When $A$ is an $M$-matrix, Alanelli and Hadjidimosin [1] considered the preconditioner $P=Q+S$, where $Q=$ $\operatorname{diag}\left(L_{11}^{-1}, I_{22}, \ldots, I_{m m}\right)$ and $S$ is given by

$$
S=\left(\begin{array}{cccc}
O_{11} & O_{12} & \cdots & O_{1 m} \\
-A_{21} L_{11}^{-1} & O_{22} & \cdots & O_{2 m} \\
\vdots & \vdots & \ddots & \vdots \\
-A_{m 1} L_{11}^{-1} & O_{m 2} & \cdots & O_{m m}
\end{array}\right)
$$

with $L_{11}$ being the lower triangular matrix in the LU triangular decomposition of $A_{11}$.

We consider the preconditioner $P_{1}=I+S_{1}$, where

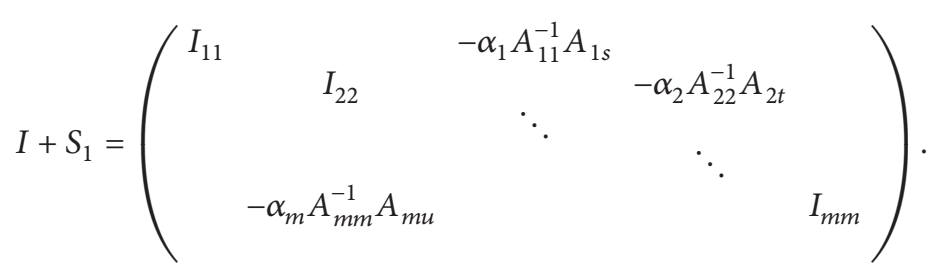

Let

$$
P_{1} A=\left(I+S_{1}\right)(D-L-U)=\widetilde{D}-\widetilde{L}-\widetilde{U},
$$

where $\widetilde{D},-\widetilde{L}$ and $-\widetilde{U}$ are block diagonally, strictly block lower, and strictly block upper triangular parts of $P_{1} A$, respectively. If $\widetilde{D}$ is nonsingular, then $(\widetilde{D}-\omega \widetilde{L})^{-1}$ and $(\widetilde{D}-\omega \widetilde{U})^{-1}$ exist and it is possible to define the SSOR iteration matrix for $P_{1} A$. Namely,

$$
\begin{aligned}
\widetilde{\mathscr{L}}_{\omega}= & (\widetilde{D}-\omega \widetilde{U})^{-1} \widetilde{D}(\widetilde{D}-\omega \widetilde{L})^{-1} \\
& \times((1-\omega) \widetilde{D}+\omega \widetilde{L}) \widetilde{D}^{-1}((1-\omega) \widetilde{D}+\omega \widetilde{U}) .
\end{aligned}
$$

Alanelli and Hadjidimos in [1] showed that the preconditioned Gauss-Seidel, the preconditioned SOR, and the preconditioned Jacobi methods with preconditioner $P$ are better than original methods. Our work in the presentation is to prove convergence of the block preconditioned SSOR

method with preconditioner $P_{1}$ and give some comparison results of the spectral radius for the case when $A$ is an $H$ matrix.

Let $|A|$ denote the matrix whose elements are the moduli of the elements of the given matrix. We call $\langle A\rangle=\left(\bar{a}_{i j}\right)$ to comparison matrix if $\bar{a}_{i j}=\left|a_{i j}\right|$ for $i=j$, if $\bar{a}_{i j}=-\left|a_{i j}\right|$ for $i \neq j$. For (4), under the previous definition, we have

$$
\langle A\rangle=\left(\begin{array}{cccc}
\left\langle A_{11}\right\rangle & -\left|A_{12}\right| & \cdots & -\mid A_{1 m} \\
-\left|A_{21}\right| & \left\langle A_{22}\right\rangle & \cdots & -\left|A_{2 m}\right| \\
\vdots & \vdots & \ddots & \vdots \\
-\left|A_{m 1}\right| & -\left|A_{m 2}\right| & \cdots & \left\langle A_{m m}\right\rangle
\end{array}\right)
$$

Let $\langle A\rangle=\langle D\rangle-|L|-|U|$, where $\langle D\rangle,-|L|$, and $-|U|$ are block diagonally, strictly block lower, and strictly block upper triangular parts of $\langle A\rangle$, respectively.

Notice that the preconditioner of the matrix $\langle A\rangle$ corresponding to $P_{1}$ is $P_{2}=I+S_{2}$; namely,




Let $P_{2}\langle A\rangle=\left(I+S_{2}\right)\langle A\rangle=\bar{D}-\bar{L}-\bar{U}$, where $\bar{D},-\bar{L}$, and $-\bar{U}$ are block diagonally, strictly block lower, and strictly block upper triangular parts of $P_{2}\langle A\rangle$, respectively.

If $\bar{D}$ is nonsingular, then $(\bar{D}-\omega \bar{L})^{-1}$ and $(\bar{D}-\omega \bar{U})^{-1}$ exist and the SSOR iteration matrix for $P_{2}\langle A\rangle$ is as follows:

$$
\begin{aligned}
\overline{\mathscr{L}}_{\omega}= & (\bar{D}-\omega \bar{U})^{-1} \bar{D}(\bar{D}-\omega \bar{L})^{-1} \\
& \times((1-\omega) \bar{D}+\omega \bar{L}) \bar{D}^{-1}((1-\omega) \bar{D}+\omega \bar{U}) .
\end{aligned}
$$

\section{Preliminaries}

A matrix $A$ is called nonnegative (positive) if each entry of $A$ is nonnegative (positive). We denote it by $A \geq 0(A>0)$. Similarly, for $n$-dimensional vector $x$, we can also define $x \geq 0$ $(x>0)$. Additionally, we denote the spectral radius of $A$ by $\rho(A)$. $A^{T}$ denotes the transpose of $A$. A matrix $A=\left(a_{i j}\right)$ is called a $Z$-matrix if for any $i \neq j, a_{i j} \leq 0$. A $Z$-matrix is a nonsingular $M$-matrix if $A$ is nonsingular and $A^{-1} \geq 0$, If $\langle A\rangle$ is a nonsingular $M$-matrix, then $A$ is called an $H$-matrix. $A=M-N$ is said to be a splitting of $A$ if $M$ is nonsingular, $A=M-N$ is said to be regular if $M^{-1} \geq 0$ and $N \geq 0$, and weak regular if $M^{-1} \geq 0$ and $M^{-1} N \geq 0$, respectively.

Some basic properties on special matrices introduced previously are given to be used in this paper.

Lemma 1 (see [2]). Let A be a Z-matrix. Then the following statements are equivalent.

(a) $A$ is an $M$-matrix.

(b) There is a positive vector $x$ such that $A x>0$.

(c) $A^{-1} \geq 0$.

(d) All principal submatrices of $A$ are $M$-matrices.

(e) All principal minors are positive.

Lemma 2 (see $[3,4])$. Let $A$ be an $M$-matrix and let $A=M-$ $N$ be a weak regular splitting. Then $\rho\left(M^{-1} N\right)<1$.

Lemma 3 (see [2]). Let $A$ and $B$ be two $n \times n$ matrices with $0 \leq B \leq A$. Then $\rho(B) \leq \rho(A)$.

Lemma 4 (see [5]). If $A$ is an H-matrix, then $\left|A^{-1}\right| \leq\langle A\rangle^{-1}$.

Lemma 5 (see [6]). Suppose that $A_{1}=M_{1}-N_{1}$ and $A_{2}=$ $M_{2}-N_{2}$ are weak regular splitting of monotone matrices $A_{1}$ and $A_{2}$, respectively, such that $M_{2}^{-1} \geq M_{1}^{-1}$. If there exists a positive vector $x$ such that $0 \leq A_{1} x \leq A_{2} x$, then for the monotone norm associated with $x$,

$$
\left\|M_{1}^{-1} N_{1}\right\|_{x} \leq\left\|M_{2}^{-1} N_{2}\right\|_{x} .
$$

In particular, if $M_{1}^{-1} N_{1}$ has a positive Perron vector, then

$$
\rho\left(M_{1}^{-1} N_{1}\right) \leq \rho\left(M_{2}^{-1} N_{2}\right) .
$$

Moreover if $x$ is a Perron vector of $M_{1}^{-1} N_{1}$ and strict inequality holds in (18), then strict inequality holds in (19).
Lemma 6. If $A$ and $B$ are two $n \times n$ matrices, then $\langle A-B\rangle \geq$ $\langle A\rangle-|B|$.

Proof. It is easy to see that $\left|a_{i j}-b_{i j}\right| \geq\left|a_{i j}\right|-\left|b_{i j}\right|$, for $i=j$, and $-\left|a_{i j}-b_{i j}\right| \geq-\left|a_{i j}\right|-\left|b_{i j}\right|$, for $i \neq j$. Therefore, $\langle A-B\rangle \geq\langle A\rangle-|B|$ is true.

Lemma 7. If $A$ is an $H$-matrix with unit diagonal elements, then $\left\|\langle A\rangle^{-1}\right\|_{\infty}>1$.

Proof. Let $\langle A\rangle=I-B$, from $\langle A\rangle$ being an $M$-matrix; then $B \geq 0$ and $\rho(B)<1$, and thus, we have

$$
\langle A\rangle^{-1}=\sum_{K=0}^{\infty} B^{k} \geq I
$$

and then $\left\|\langle A\rangle^{-1}\right\|_{\infty}>1$.

\section{Convergence Results}

Let $e_{i}=(1, \ldots, 1)^{T} \in R^{n_{i}}, i=1,2, \ldots, m, e=\left(e_{1}^{T}, \ldots, e_{m}^{T}\right)^{T}$, $r=\left(r_{1}^{T}, \ldots, r_{m}^{T}\right)^{T}=\langle A\rangle^{-1} e, O_{i}=(0, \ldots, 0)^{T} \in R^{n_{i}}$, where $r$ and $e$ are partitioned in accordance with the block partitioning of the matrix $A$, and let

$$
\begin{array}{r}
s_{i}=\left\|\left\langle A_{i i}\right\rangle^{-1}\left|A_{i k}\right| e_{k}\right\|_{\infty}, \\
h_{i}=\frac{1}{s_{i}\left(2\left\|\langle A\rangle^{-1}\right\|_{\infty}-1\right)}, \\
i=1,2, \ldots, m .
\end{array}
$$

Theorem 8. Let $A$ be a nonsingular H-matrix; if $\left|\alpha_{i}\right|<h_{i}$, $i=1,2, \ldots, m$, then $P_{1} A$ is also an $H$-matrix.

Proof. From $A$ being an $H$-matrix, we have $r>0$, and $r_{k} \leq \|$ $\langle A\rangle^{-1} \|_{\infty} e_{k}$. Let

$$
\begin{aligned}
& \left(\left(P_{1} A\right)_{i j}\right) \\
& \quad= \begin{cases}A_{i j}-\alpha_{i} A_{i i}^{-1} A_{i k} A_{k j}, & i \neq j, i, j=1,2, \ldots, m, k \neq i, \\
A_{i i}-\alpha_{i} A_{i i}^{-1} A_{i k} A_{k i}, & i=j, i, j=1,2, \ldots, m, k \neq i .\end{cases}
\end{aligned}
$$

Then

$$
\begin{aligned}
\left(\left\langle P_{1} A\right\rangle r\right)_{i}= & \left\langle A_{i i}-\alpha_{i} A_{i i}^{-1} A_{i k} A_{k i}\right\rangle r_{i} \\
& -\sum_{j \neq i, k}^{m}\left|A_{i j}-\alpha_{i} A_{i i}^{-1} A_{i k} A_{k j}\right| r_{j} \\
& -\left|A_{i k}-\alpha_{i} A_{i i}^{-1} A_{i k} A_{k k}\right| r_{k} \\
\geq & \left\langle A_{i i}\right\rangle r_{i}-\left|\alpha_{i}\right|\left|A_{i i}^{-1}\right|\left|A_{i k}\right|\left|A_{k i}\right| r_{i}-\sum_{j \neq i, k}^{m}\left|A_{i j}\right| \\
& -\sum_{j \neq i, k}^{m}\left|\alpha_{i}\right|\left|A_{i i}^{-1}\right|\left|A_{i k}\right|\left|A_{k j}\right| r_{j}
\end{aligned}
$$




$$
\begin{aligned}
& -\left|A_{i k}\right| r_{k}-\left|\alpha_{i}\right|\left|A_{i i}^{-1}\right|\left|A_{i k}\right|\left|A_{k k}\right| r_{k} \\
& \geq e_{i}-\left|\alpha_{i}\right|\left\langle A_{i i}\right\rangle^{-1}\left|A_{i k}\right|\left|A_{k i}\right| r_{i} \\
& -\sum_{j \neq i, k}^{m}\left|\alpha_{i}\right|\left\langle A_{i i}\right\rangle^{-1}\left|A_{i k}\right|\left|A_{k j}\right| r_{j} \\
& -\left|\alpha_{i}\right|\left\langle A_{i i}\right\rangle^{-1}\left|A_{i k}\right|\left|A_{k k}\right| r_{k} \\
& =e_{i}+\left|\alpha_{i}\right|\left\langle A_{i i}\right\rangle^{-1}\left|A_{i k}\right| \\
& \times\left(-\sum_{j \neq k}^{m}\left|A_{k j}\right| r_{j}-\left|A_{k k}\right| r_{k}\right. \\
& \left.+\left\langle A_{k k}\right\rangle r_{k}-\left\langle A_{k k}\right\rangle r_{k}\right) \\
& =e_{i}+\left|\alpha_{i}\right|\left\langle A_{i i}\right\rangle^{-1}\left|A_{i k}\right|\left(e_{k}-2 r_{k}\right) \\
& \geq e_{i}-\left|\alpha_{i}\right|\left(2\left\|\langle A\rangle^{-1}\right\|_{\infty}-1\right)\left\langle A_{i i}\right\rangle^{-1}\left|A_{i k}\right| e_{k} \\
& \geq e_{i}-\left|\alpha_{i}\right| s_{i}\left(2\left\|\langle A\rangle^{-1}\right\|_{\infty}-1\right) e_{i} \\
& >\mathrm{O}_{i} \text {. }
\end{aligned}
$$

Therefore, $\left\langle P_{1} A\right\rangle$ is an $M$-matrix, and then $P_{1} A$ is an $H$ matrix.

Theorem 9. If $A$ is a nonsingular $H$-matrix with unit diagonal elements, $0<\omega \leq 1$ and $\left|\alpha_{i}\right|<h_{i}, i=1,2, \ldots, m$. Then $\rho\left(\widetilde{\mathscr{L}}_{\omega}\right)<1$.

Proof. From Theorem 8, we know $\left\langle P_{1} A\right\rangle=\langle\widetilde{D}\rangle-|\widetilde{L}|-|\widetilde{U}|$ is an $M$-matrix; if we let

$$
\begin{aligned}
\left\langle P_{1} A\right\rangle= & \frac{1}{\omega(2-\omega)}(\langle\widetilde{D}\rangle-\omega|\widetilde{L}|)\langle\widetilde{D}\rangle^{-1}(\langle\widetilde{D}\rangle-\omega|\widetilde{U}|) \\
& -\frac{1}{\omega(2-\omega)}((1-\omega)\langle\widetilde{D}\rangle+\omega|\widetilde{L}|)\langle\widetilde{D}\rangle^{-1} \\
& \times((1-\omega)\langle\widetilde{D}\rangle+\omega|\widetilde{U}|),
\end{aligned}
$$

then the SSOR iteration matrix for $\left\langle P_{1} A\right\rangle$ is as follows:

$$
\begin{aligned}
\ddot{\mathscr{L}}_{\omega}= & (\langle\widetilde{D}\rangle-\omega|\widetilde{U}|)^{-1}\langle\widetilde{D}\rangle(\langle\widetilde{D}\rangle-\omega|\widetilde{L}|)^{-1} \\
& \times((1-\omega)\langle\widetilde{D}\rangle+\omega|\widetilde{L}|)\langle\widetilde{D}\rangle^{-1} \\
& \times((1-\omega)\langle\widetilde{D}\rangle+\omega|\widetilde{U}|) .
\end{aligned}
$$

Since $\left\langle P_{1} A\right\rangle$ is an $M$-matrix; we have $\langle\widetilde{D}\rangle,\langle\widetilde{D}\rangle-\omega|\widetilde{L}|$ and $\langle\widetilde{D}\rangle-\omega|\widetilde{U}|$ are $M$-matrices; by simple calculation, we obtain that (24) is a weak regular splitting; from Lemma 2, we know that $\rho\left(\ddot{\mathscr{L}}_{\omega}\right)<1$. Since

$$
\begin{aligned}
\left|\widetilde{\mathscr{L}}_{\omega}\right|= & \mid(\widetilde{D}-\omega \widetilde{U})^{-1} D(\widetilde{D}-\omega \widetilde{L})^{-1} \\
& \times((1-\omega) \widetilde{D}+\omega \widetilde{L}) D^{-1}((1-\omega) \widetilde{D}+\omega \widetilde{U}) \mid \\
= & \mid\left(I-\omega \widetilde{D}^{-1} \widetilde{U}\right)^{-1}\left(I-\omega \widetilde{D}^{-1} \widetilde{L}\right)^{-1} \\
& \times\left((1-\omega) I+\omega D^{-1} \widetilde{L}\right)\left((1-\omega) I+\omega \widetilde{D}^{-1} \widetilde{U}\right) \mid \\
\leq & \left|\left(I-\omega \widetilde{D}^{-1} \widetilde{U}\right)^{-1}\right|\left|\left(I-\omega \widetilde{D}^{-1} \widetilde{L}\right)^{-1}\right| \\
& \times\left|\left((1-\omega) I+\omega D^{-1} \widetilde{L}\right)\right|\left|\left((1-\omega) I+\omega \widetilde{D}^{-1} \widetilde{U}\right)\right| \\
\leq & \left(I-\omega\langle\widetilde{D}\rangle^{-1}|\widetilde{U}|\right)^{-1}\left(I-\omega\langle\widetilde{D}\rangle^{-1}|\widetilde{L}|\right)^{-1} \\
& \times\left((1-\omega) I+\omega\langle\widetilde{D}\rangle^{-1}|\widetilde{L}|\right)\left((1-\omega) I+\omega\langle\widetilde{D}\rangle^{-1}|\widetilde{U}|\right) \\
= & \rho\left(\ddot{\mathscr{L}} \omega_{\omega}\right.
\end{aligned}
$$

then, by Lemma 3, $\rho\left(\widetilde{\mathscr{L}}_{\omega}\right) \leq \rho\left(\left|\widetilde{\mathscr{L}}_{\omega}\right|\right) \leq \rho\left(\ddot{\mathscr{L}}_{\omega}\right)<1$.

\section{Comparison Results of Spectral Radius}

Theorem 10. Let $A$ be a nonsingular $H$-matrix with unit diagonal elements, $0<\omega \leq 1$ and $\left|\alpha_{i}\right|<h_{i}, i=1,2, \ldots, m$. Then $P_{2}\langle A\rangle$ is an M-matrix and $\rho\left(\overline{\mathscr{L}}_{\omega}\right)<1$.

Proof. Similar to the proof of Theorems 8 and 9 , it is easy to get the proof of this theorem.

In what follows we will give some comparison results on the spectral radius of preconditioned SSOR iteration matrices with different preconditioner.

Let

$$
\begin{aligned}
\langle A\rangle= & \widehat{M}-\widehat{N} \\
= & \frac{1}{\omega(2-\omega)}(\langle D\rangle-\omega|L|)\langle D\rangle^{-1}(\langle D\rangle-\omega|U|) \\
& -\frac{1}{\omega(2-\omega)}((1-\omega)\langle D\rangle+\omega|L|)\langle D\rangle^{-1} \\
& \times((1-\omega)\langle D\rangle+\omega|U|),
\end{aligned}
$$

where

$$
\begin{aligned}
\widehat{M}= & \frac{1}{\omega(2-\omega)}(\langle D\rangle-\omega|L|)\langle D\rangle^{-1}(\langle D\rangle-\omega|U|), \\
\widehat{N}= & \frac{1}{\omega(2-\omega)}((1-\omega)\langle D\rangle+\omega|L|)\langle D\rangle^{-1} \\
& \quad \times((1-\omega)\langle D\rangle+\omega|U|) .
\end{aligned}
$$


Then the SSOR iteration matrix for $\langle A\rangle$ is as follows:

$$
\begin{aligned}
\widehat{\mathscr{L}}_{\omega}= & \widehat{M}^{-1} \widehat{N} \\
= & (\langle D\rangle-\omega|U|)^{-1}\langle D\rangle(\langle D\rangle-\omega|L|)^{-1} \\
& \times((1-\omega)\langle D\rangle+\omega|L|)\langle D\rangle^{-1}((1-\omega)\langle D\rangle+\omega|U|),
\end{aligned}
$$

and let

$$
\begin{aligned}
P_{2}\langle A\rangle= & \bar{M}-\bar{N} \\
= & \frac{1}{\omega(2-\omega)}(\bar{D}-\omega \bar{L}) \bar{D}^{-1}(\bar{D}-\omega \bar{U}) \\
& -\frac{1}{\omega(2-\omega)}((1-\omega) \bar{D}+\omega \bar{L}) \bar{D}^{-1} \\
& \times((1-\omega) \bar{D}+\omega \bar{U}),
\end{aligned}
$$

where

$$
\begin{gathered}
\bar{M}=\frac{1}{\omega(2-\omega)}(\bar{D}-\omega \bar{L}) \bar{D}^{-1}(\bar{D}-\omega \bar{U}) \\
\bar{N}=\frac{1}{\omega(2-\omega)}((1-\omega) \bar{D}+\omega \bar{L}) \bar{D}^{-1}((1-\omega) \bar{D}+\omega \bar{U}) .
\end{gathered}
$$

Then the AOR iteration matrix for $P_{2}\langle A\rangle$ is (17).

Theorem 11. If $A$ is a nonsingular $H$-matrix with unit diagonal elements, $0<\omega \leq 1$ and $\left|\alpha_{i}\right|<h_{i}, i=1,2, \ldots, m$. Then $\rho\left(\overline{\mathscr{L}}_{\omega}\right) \leq \rho\left(\widehat{\mathscr{L}}_{\omega}\right)$.

Proof. Since $\langle A\rangle$ is a nonsingular $M$-matrix, by Theorem 10 , $P_{2}\langle A\rangle$ is a nonsingular $M$-matrix, and thus $\langle A\rangle$ and $P_{2}\langle A\rangle$ are two monotone matrices.

From $\langle A\rangle$ and $P_{2}\langle A\rangle$ being $M$-matrices, we can get $\langle D\rangle$, $\bar{D}, \widehat{M}$, and $\bar{M}$ are $M$-matrices, together with

$$
\begin{gathered}
\left((1-\omega) I+\omega\langle D\rangle^{-1}|L|\right)\langle D\rangle^{-1}\left((1-\omega) I+\omega\langle D\rangle^{-1}|U|\right)>0 \\
\left((1-\omega) I+\omega \bar{D}^{-1} \bar{L}\right) \bar{D}^{-1}\left((1-\omega) I+\omega \bar{D}^{-1} \bar{U}\right)>0
\end{gathered}
$$

We obtain that $\langle A\rangle=\widehat{M}-\widehat{N}$ and $P_{2}\langle A\rangle=\bar{M}-\bar{N}$ are two weak regular splittings. By simple calculation, we have

$$
\begin{aligned}
\bar{M} & =\frac{1}{\omega(2-\omega)}(\bar{D}-\omega \bar{L}) \bar{D}^{-1}(\bar{D}-\omega \bar{U}) \\
& \leq \frac{1}{\omega(2-\omega)}(\langle D\rangle-\omega|L|)\langle D\rangle^{-1}(\langle D\rangle-\omega|U|)=\widehat{M}
\end{aligned}
$$

and thus $\bar{M}^{-1} \geq \widehat{M}^{-1} \geq 0$; letting $x=\langle A\rangle^{-1} e>0$, then $\left(P_{2}\langle A\rangle-\langle A\rangle\right) x=\left(I+S_{2}\right) e>0$; since $\bar{M}^{-1} \geq \widehat{M}^{-1} \geq 0$, we have

$\bar{M}^{-1}\left(P_{2}\langle A\rangle\right) x=\left(I-\bar{M}^{-1} \bar{N}\right) x \geq \widehat{M}^{-1}\langle A\rangle x=\left(I-\widehat{M}^{-1} \widehat{N}\right) x$.
It follows that

$$
\left\|\bar{M}^{-1} \bar{N}\right\|_{x} \leq\left\|\widehat{M}^{-1} \widehat{N}\right\|_{x}
$$

As $\langle A\rangle=\widehat{M}-\widehat{N}$ is a weak regular splitting, there exists a positive perron vector $y$; by Lemma 5 , the following inequality holds:

$$
\rho\left(\bar{M}^{-1} \bar{N}\right) \leq \rho\left(\widehat{M}^{-1} \widehat{N}\right)
$$

that is,

$$
\rho\left(\overline{\mathscr{L}}_{\omega}\right) \leq \rho\left(\widehat{\mathscr{L}}_{\omega}\right)
$$

When $A$ is a nonsingular $M$-matrix, we have $A=\langle A\rangle$. If $\alpha_{i}>0, i=1,2, \ldots, m$, then $P_{2}=P_{1}$. Furthermore, we have $\overline{\mathscr{L}}_{\omega}=\widetilde{\mathscr{L}}_{\omega}$ and $\mathscr{L}_{\omega}=\widehat{\mathscr{L}}_{\omega}$; therefore, we get the following result.

Corollary 12. Let $A$ be a nonsingular $M$-matrix with unit diagonal elements, $0<\alpha_{i}<\left|h_{i}\right|, i=1,2, \ldots, m$, and $0<$ $\omega \leq 1$. Then

$$
\rho\left(\overline{\mathscr{L}}_{\omega}\right)=\rho\left(\widetilde{\mathscr{L}}_{\omega}\right) \leq \rho\left(\widehat{\mathscr{L}}_{\omega}\right)=\rho\left(\mathscr{L}_{\omega}\right) .
$$

Theorem 13. Let $A$ be a nonsingular $H$-matrix with unit diagonal elements, $0<\omega \leq 1$ and $\left|\alpha_{i}\right|<h_{i}, i=1,2, \ldots, m$. Then $\rho\left(\ddot{\mathscr{L}}_{\omega}\right) \leq \rho\left(\overline{\mathscr{L}}_{\omega}\right)$.

Proof. Let

$$
\begin{aligned}
\left\langle P_{1} A\right\rangle= & \frac{1}{\omega(2-\omega)}(\langle\widetilde{D}\rangle-\omega|\widetilde{L}|)\langle\widetilde{D}\rangle^{-1}(\langle\widetilde{D}\rangle-\omega|\widetilde{U}|) \\
& -\frac{1}{\omega(2-\omega)}((1-\omega)\langle\widetilde{D}\rangle+\omega|\widetilde{L}|)\langle\widetilde{D}\rangle^{-1} \\
& \times((1-\omega)\langle\widetilde{D}\rangle+\omega|\widetilde{U}|) .
\end{aligned}
$$

Then the SSOR iteration matrix for $\left\langle P_{1} A\right\rangle$ is $\ddot{\mathscr{L}}_{\omega}$ which is defined in the proof of Theorem 9 , and let

$$
\begin{aligned}
P_{2}\langle A\rangle= & \frac{1}{\omega(2-\omega)}(\bar{D}-\omega \bar{L}) \bar{D}^{-1}(\bar{D}-\omega \bar{U}) \\
& -\frac{1}{\omega(2-\omega)}((1-\omega) \bar{D}+\omega \bar{L}) \bar{D}^{-1} \\
& \times((1-\omega) \bar{D}+\omega \bar{U}) .
\end{aligned}
$$

Then the SSOR iteration matrix for $P_{2}\langle A\rangle$ is (17). It is easy to know that the previous two splittings are weak regular splittings. Furthermore, by Lemma 6, we have the following result, for any $i, i=1,2, \ldots, m$,

$$
\begin{aligned}
\left\langle\widetilde{D}_{i i}\right\rangle & =\left\langle A_{i i}-\alpha_{i} A_{i i}^{-1} A_{i k} A_{k i}\right\rangle \\
& \geq\left\langle A_{i i}\right\rangle-\left|\alpha_{i}\right|\left\langle A_{i i}\right\rangle^{-1}\left|A_{i k}\right|\left|A_{k i}\right|=\left\langle\bar{D}_{i i}\right\rangle .
\end{aligned}
$$

From $\left\langle P_{1} A\right\rangle$ and $P_{2}\langle A\rangle$ being two $M$-matrices, we have

$$
0 \leq\langle\widetilde{D}\rangle^{-1} \leq \bar{D}^{-1}
$$


TABLE 1: Comparison of spectral radius with preconditioner $P_{1}$.

\begin{tabular}{|c|c|c|c|c|c|c|}
\hline$\omega, r$ & $N$ & $\rho\left(\widetilde{\mathscr{L}}_{\omega}\right)$ & $\rho\left(\overline{\mathscr{L}}_{\omega}\right)$ & $\rho\left(\ddot{\mathscr{L}}_{\omega}\right)$ & $\rho\left(\widehat{\mathscr{L}}_{\omega}\right)$ & $\rho\left(\mathscr{L}_{\omega}\right)$ \\
\hline \multirow{3}{*}{$\omega=0.8$} & 100 & 0.5636 & 0.8635 & 0.7404 & 0.8999 & 0.6288 \\
\hline & 200 & 0.6030 & 0.9195 & 0.7698 & 0.9473 & 0.7059 \\
\hline & 500 & 0.6120 & 0.9751 & 0.7844 & 0.9847 & 0.7103 \\
\hline \multirow{3}{*}{$\omega=0.6$} & 100 & 0.3650 & 0.7906 & 0.5923 & 0.8530 & 0.4770 \\
\hline & 200 & 0.4510 & 0.8832 & 0.6507 & 0.9240 & 0.5769 \\
\hline & 500 & 0.4345 & 0.9730 & 0.6766 & 0.9835 & 0.5609 \\
\hline \multirow{4}{*}{$\omega=0.9$} & 100 & 0.2387 & 0.7512 & 0.6647 & 0.8087 & 0.3602 \\
\hline & 200 & 0.3494 & 0.8491 & 0.5800 & 0.9009 & 0.4899 \\
\hline & 500 & 0.3569 & 0.9561 & 0.6284 & 0.9731 & 0.5019 \\
\hline & 1000 & 0.3674 & 0.9738 & 0.6316 & 0.9840 & 0.5173 \\
\hline
\end{tabular}

and then

$$
\begin{aligned}
\ddot{\mathscr{L}}_{\omega}= & (\langle\widetilde{D}\rangle-\omega|\widetilde{U}|)^{-1}\langle\widetilde{D}\rangle(\langle\widetilde{D}\rangle-\omega|\widetilde{L}|)^{-1} \\
& \times((1-\omega)\langle\widetilde{D}\rangle+\omega|\widetilde{L}|)\langle\widetilde{D}\rangle^{-1}((1-\omega)\langle\widetilde{D}\rangle+\omega|\widetilde{U}|) \\
= & \left(I-\omega\langle\widetilde{D}\rangle^{-1}|\widetilde{U}|\right)^{-1}\left(I-\omega\langle\widetilde{D}\rangle^{-1}|\widetilde{L}|\right)^{-1} \\
& \times\left((1-\omega) I+\omega\langle\widetilde{D}\rangle^{-1}|\widetilde{L}|\right)\left((1-\omega) I+\omega\langle\widetilde{D}\rangle^{-1}|\widetilde{U}|\right) \\
\leq & \left(I-\omega \bar{D}^{-1}|\widetilde{U}|\right)^{-1}\left(I-\omega \bar{D}^{-1}|\widetilde{L}|\right)^{-1} \\
& \times\left((1-\omega) I+\omega \bar{D}^{-1}|\widetilde{L}|\right)\left((1-\omega) I+\omega \bar{D}^{-1}|\widetilde{U}|\right) \\
= & \overline{\mathscr{L}}_{\omega} .
\end{aligned}
$$

Therefore, by Lemma 3, $\rho\left(\ddot{\mathscr{L}}_{\omega}\right) \leq \rho\left(\overline{\mathscr{L}}_{\omega}\right)$.
Combining the previous Theorems, we can obtain the following conclusion.

Theorem 14. Let $A$ be a nonsingular $H$-matrix with unit diagonal elements, $0<\omega \leq 1$ and $\left|\alpha_{i}\right|<h_{i}, i=1,2, \ldots, m$. Then

$$
\rho\left(\widetilde{\mathscr{L}}_{\omega}\right) \leq \rho\left(\ddot{\mathscr{L}}_{\omega}\right) \leq \rho\left(\overline{\mathscr{L}}_{\omega}\right) \leq \rho\left(\widehat{\mathscr{L}}_{\omega}\right)<1 .
$$

\section{Numerical Example}

For randomly generated nonsingular $H$-matrices for $n=$ $100,200,500,1000$ with $n_{1}=n_{2}=\cdots=n_{m}=5$, we have determined the spectral radius of the iteration matrices of SSOR method mentioned previously with preconditioner $P_{1}$. We report the spectral radius of the corresponding iteration matrix by $\rho$. The $m$ parameters $\alpha_{i}, i=1,2, \ldots, m$, are taken from the $m$ equal-partitioned points of the interval $[0,1]$. We take

$$
P_{1}=\left(\begin{array}{ccccc}
A_{11}^{-1} & A_{11}^{-1} A_{12} & O_{13} & \cdots & O_{1 m} \\
O_{21} & A_{22}^{-1} & A_{11}^{-1} A_{23} & \ddots & \vdots \\
\vdots & \ddots & \ddots & \ddots & \vdots \\
O_{m-1,1} & \cdots & O_{m-1, m-2} & A_{m-1, m-1}^{-1} & A_{m-1, m-1}^{-1} A_{m-1, m} \\
A_{m m}^{-1} A_{m 1} & O_{m 2} & \cdots & O_{m, m-1} & A_{m m}^{-1}
\end{array}\right)
$$

For $P_{1}$, we make two groups of experiments. In Figure 1, we test the relation between $\omega$ and $\rho$, when $N=100, \omega=0.6$, where " $\times$ ", " +", “*”, “." and "o" denote the spectral radius of $\langle A\rangle, P_{2}\langle A\rangle,\left\langle P_{1} A\right\rangle, A$, and $P_{1} A$, respectively. In Table 1 , the meaning of notations $\rho\left(\widetilde{\mathscr{L}}_{\omega}\right), \rho\left(\overline{\mathscr{L}}_{\omega}\right), \rho\left(\ddot{\mathscr{L}}_{\omega}\right), \rho\left(\widehat{\mathscr{L}}_{\omega}\right)$, and $\rho\left(\mathscr{L}_{\omega}\right)$ denotes the spectral radius of $P_{1} A, P_{2}\langle A\rangle,\left\langle P_{1} A\right\rangle$, $\langle A\rangle$, and $A$, respectively.

From Figure 1 and Table 1, we can conclude that the spectral radius of the preconditioned SSOR method with preconditioner $P_{1}$ is the best among others, which further illustrates that, Theorem 14 is true.

\section{Acknowledgments}

The authors express their thanks to the editor Professor HakKeung Lam and the anonymous referees who made much useful and detailed suggestions that helped them to correct some minor errors and improve the quality of the paper. 


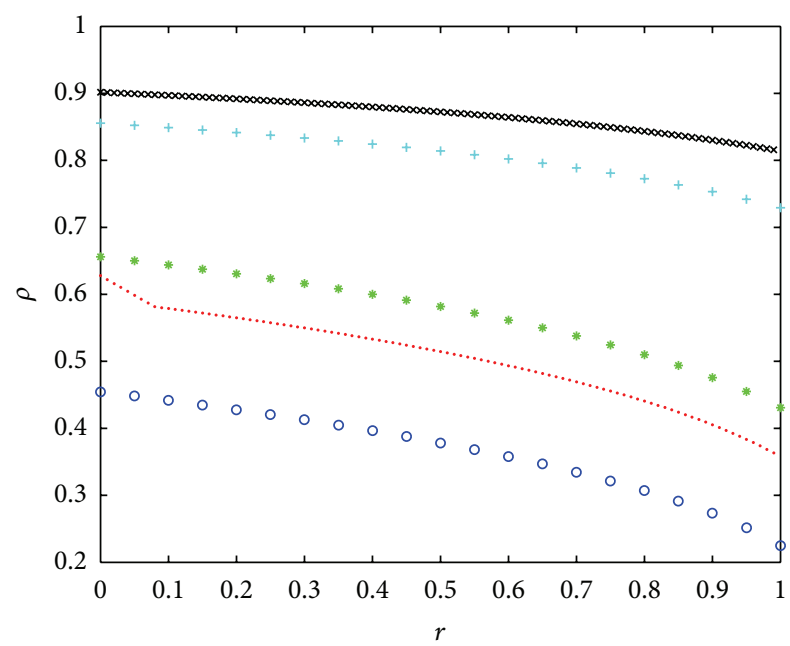

FIgURE 1: The relation between $\omega$ and $\rho$, when $N=100, \omega=0.6$.

\section{References}

[1] M. Alanelli and A. Hadjidimos, "Block Gauss elimination followed by a classical iterative method for the solution of linear systems," Journal of Computational and Applied Mathematics, vol. 163, no. 2, pp. 381-400, 2004.

[2] A. Berman and R. J. Plemmons, Nonnegative Matrices in the Mathematical Sciences, vol. 9 of Classics in Applied Mathematics, Society for Industrial and Applied Mathematics (SIAM), Philadelphia, Pa, USA, 1994.

[3] W. Li and Z.-y. You, "The multi-parameters overrelaxation method," Journal of Computational Mathematics, vol. 16, no. 4, pp. 367-374, 1998.

[4] R. S. Varga, Matrix Iterative Analysis, Prentice-Hall, Englewood Cliffs, NJ, USA, 1962.

[5] L. Yu. Kolotilina, "Two-sided bounds for the inverse of an $H$ matrix," Linear Algebra and Its Applications, vol. 225, pp. 117-123, 1995.

[6] M. Neumann and R. J. Plemmons, "Convergence of parallel multisplitting iterative methods for M-matrices," Linear Algebra and Its Applications, vol. 88-89, pp. 559-573, 1987. 


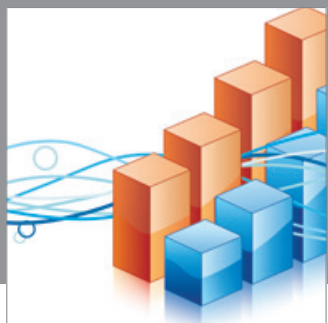

Advances in

Operations Research

mansans

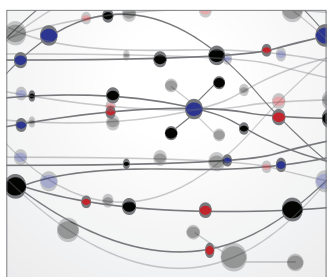

The Scientific World Journal
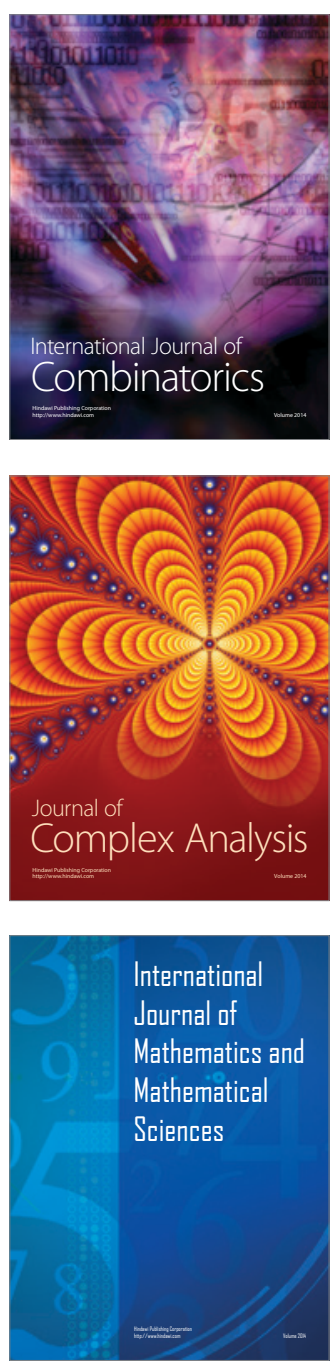


Submit your manuscripts at http://www.hindawi.com
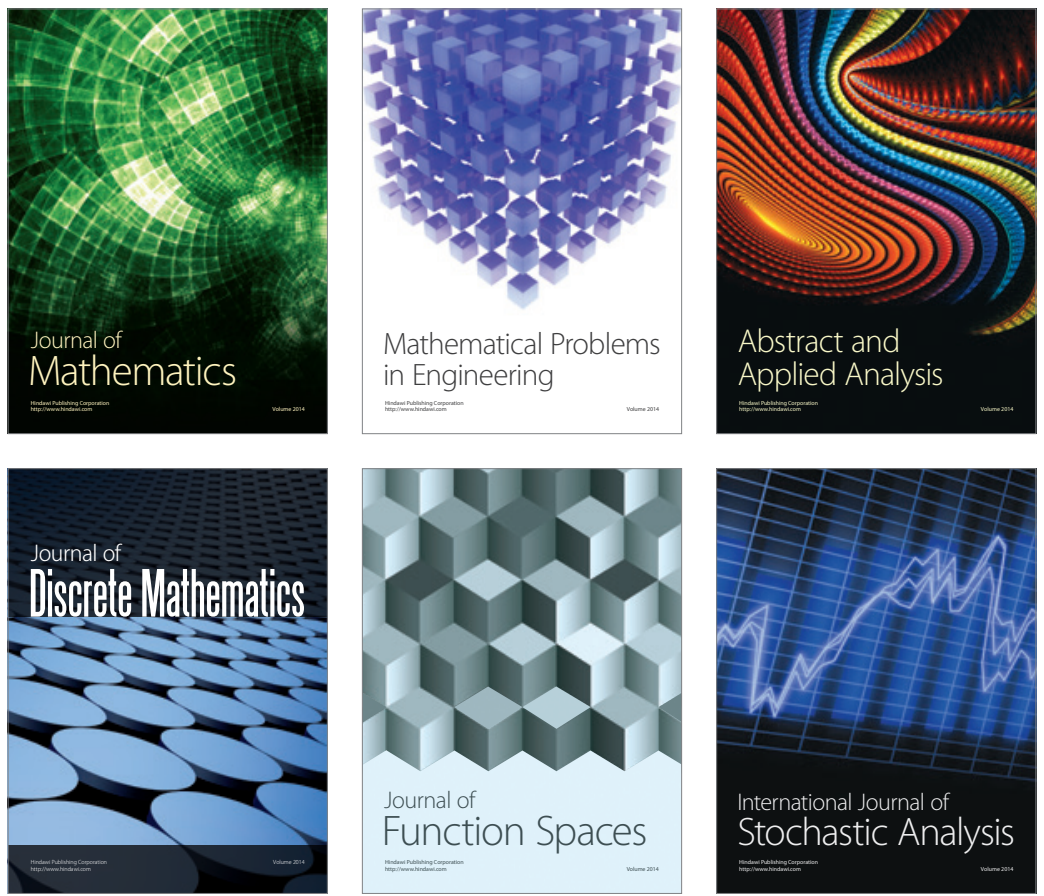

Journal of

Function Spaces

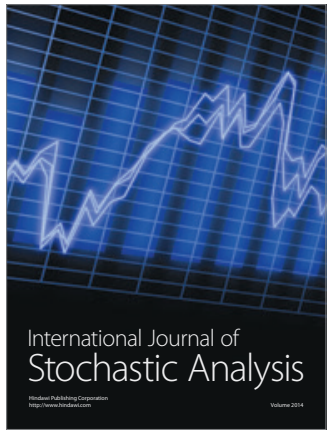

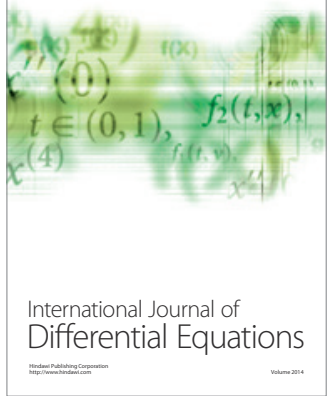
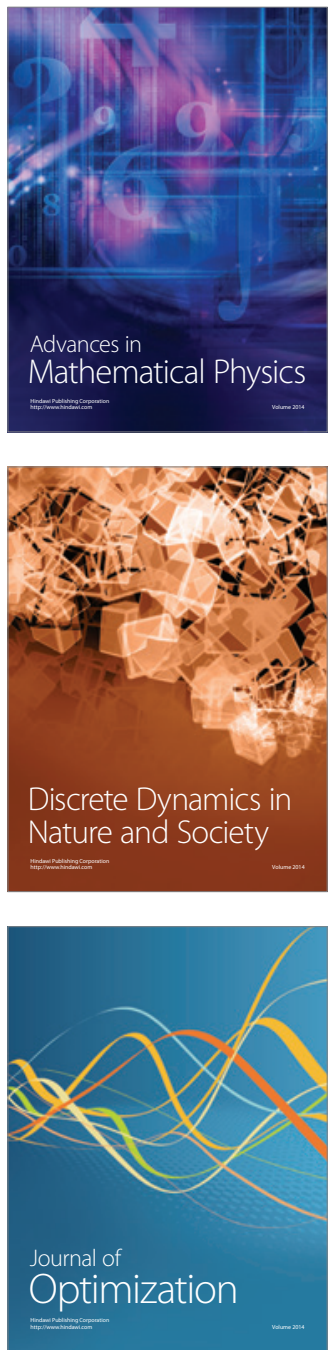